\title{
Expression of the PIM2 gene is associated with more aggressive clinical course in patients with chronic lymphocytic leukemia
}

\author{
Katarzyna Kapelko-Słowik ${ }^{1, A-F}$, Jarosław Dybko ${ }^{1, B, E}$, Krzysztof Grzymajł0 2,B-D, Bożena Jaźwiec ${ }^{1, B, C}$, \\ Donata Urbaniak-Kujda ${ }^{1, A-D}$, Mirosław Słowik ${ }^{3, B-D}$, Stanisław Potoczek ${ }^{1, B, C}$, Dariusz Wołowiec ${ }^{1, A, C, E, F}$ \\ ${ }^{1}$ Department of Hematology, Neoplastic Blood Disorders and Bone Marrow Transplantation, Wroclaw Medical University, Poland \\ 2 Department of Biochemistry, Pharmacology and Toxicology, Wrocław University of Environmental and Life Sciences, Poland \\ ${ }^{3}$ Department of Ophthalmology, Wroclaw Medical University, Poland \\ A - research concept and design; $B$ - collection and/or assembly of data; $C$ - data analysis and interpretation; \\ $D$ - writing the article; $E$ - critical revision of the article; $F$ - final approval of the article
}

Address for correspondence

Katarzyna Kapelko-Słowik

E-mail:kks9999@wp.pl

\section{Funding sources}

The study was financially supported

by the statutory funds of Wroclaw Medical

University (ST.A080.17.035).

Conflict of interest

None declared

Received on April 11, 2017

Reviewed on April 29, 2017

Accepted on May 5, 2018

Published online on December 7, 2018

\section{Abstract}

Background. The PIM2 gene belongs to the PIM family, which encodes serine/threonine kinases involved in cell survival and apoptosis. The relation between the expression of the PIM2 gene and the course of chronic lymphocytic leukemia (CLL) has not been fully determined.

Objectives. The aim of the study was to evaluate the role of the PIM2 gene as a marker of CLL malignancy and its importance as a predictive and prognostic factor.

Material and methods. Sixty-seven patients, 35 females and 32 males, aged $49-90$ years, with de novo CLL, and 14 healthy individuals were enrolled in the study. Expression of the PIM2 gene was analyzed using TagMan RQ-PCR assay and western blot test.

Results. Median PIM2 gene expression in CLL patients was higher than in controls. Patients with high expression of the PIM2 gene had shorter progression-free survival and time to first treatment than patients with low PIM2 expression. It was found that patients with CR had lower expression of the PIM2 gene than patients without complete remission (CR). Notably, associations between high PIM2 expression and rapid lymphocyte doubling time, the percentage of malignant lymphocytes with ZAP70 expression and the Rai stage were revealed.

Conclusions. We found that the PIM2 gene is associated with a more aggressive clinical course of CLL.

Key words: PIM2 gene, chronic lymphocytic leukemia, prognostic factor

Cite as

Kapelko-Słowik K, Dybko J, Grzymajło K, et al. Expression of the PIM2 gene is associated with more aggressive clinical course in patients with chronic lymphocytic leukemia. $A d v$ Clin Exp Med. 2019;28(3):385-390. doi:10.17219/acem/90771

DOI

10.17219/acem/90771

Copyright

Copyright by Author(s)

This is an article distributed under the terms of the

Creative Commons Attribution Non-Commercial License

(http://creativecommons.org/licenses/by-nc-nd/4.0/) 


\section{Introduction}

Chronic lymphocytic leukemia (CLL) is a disease strongly connected with an accumulation of neoplastic cells. Suppression of apoptosis in vivo of leukemic lymphocytes is the most characteristic feature inducing their accumulation in CLL patients. The extensive research regarding intercellular paths leading to cell life prolongation and resistance to pro-apoptotic stimuli is still in progress. In recent years, this research has led to the discovery of an antiapoptotic kinase PIM2. PIM2 kinase was identified as taking part in murine lymphoid cell transformation. ${ }^{1}$ PIM2 along with PIM1 and PIM3 belong to the serine/threonine kinase family encoded by corresponding oncogenes. ${ }^{2,3}$ PIM2 gene expression is regulated at both the mRNA and protein level by cytokines (e.g., interleukin 3 - IL-3) involved in the differentiation of hematopoietic cells. This expression has been detected in spleen thymus and in proliferating hematopoietic cells. Constitutive expression of PIM2 extends cellular lifespan and makes the cells insensitive to proapoptotic stimuli or deficiency of exogenous growth factors. ${ }^{4}$ Elevated expression of the PIM2 gene was revealed in cell lines derived from human solid (SW480, A549, G361) and hematological tumors (HL60, K562, RAIJ), acute myeloid leukemia, as well as lymphoid malignant cells. ${ }^{5,6}$ This indicates a possibility of this factor playing a significant role in neoplastic growth pathogenesis. ${ }^{7-9}$ In previous studies, increased expression of the PIM2 gene in CLL and follicular and large-cell lymphoma was found. PIM2 mRNA expression was significantly higher in CLL lymphocytes than in normal B-lymphocytes and was correlated with advanced stage as well as shorter lymphocyte doubling time (LDT) ${ }^{10}$ Therefore, the aim of this study was to further evaluate PIM2 expression in ex vivo CLL cells and to evaluate its possible predictive and prognostic value in this disease as assessed by the response to chemotherapy and survival analysis.

\section{Material and methods}

\section{Patients}

Sixty-seven patients with newly diagnosed CLL from the Department of Hematology, Wroclaw Medical University, Poland, were enrolled in the study. Among them were 35 females and 32 males, aged 49-90 years (median age was 66 years). Peripheral blood samples were collected from all patients at the time of diagnosis, whereas only samples with monoclonal lymphoid cells representing more than $80 \%$ of total cellularity were assessed. The follow-up period of the patients was 11-71 months. During that period the indications for cytostatic treatment, according to the International Workshop on Chronic Lymphocytic Leukemia (iwCLL) guidelines, appeared in all patients. ${ }^{11}$ Twenty-two patients under 60 years of age with an estimated glomerular filtration rate $>70 \mathrm{~mL} / \mathrm{min}$ and without clinically significant comorbidities received 4-6 cycles of FC-R (fludarabine, cyclophosphamide, rituximab), 33 patients received 6-9 cycles of LP (chlorambucil, prednisone) and 12 patients received 4-6 cycles of RB (rituximab, bendamustine). Complete remission (CR) was assessed according to the standard criteria. ${ }^{12}$ Thirty-two patients reached CR and 11 patients partial remission (PR). Thirteen patients reached CR after FC-R therapy, and 10 patients after LP or RB. In 16 patients peripheral blood lymphocytes were additionally analyzed in CR following the chemotherapy. The control group consisted of 14 healthy individuals, patients of the Department of Ophthalmology, Wroclaw Medical University, matched by age and sex to the examined group. The clinical data of CLL patients and controls is presented in Table 1.

\section{Methods}

Peripheral blood was obtained from the patients at the time of diagnosis and control subjects by venipuncture. Mononuclear cells (MNC) were separated by centrifugation over Gradisol L lymphocyte separation medium (Aqua Medica, Warszawa, Poland). The isolated cells were stored at $-80^{\circ} \mathrm{C}$ as dry pellets.

\section{Real-time PCR}

Total RNA was isolated from $6 \times 10^{6} \mathrm{MNC}$ using TriRaegent ${ }^{\circledR}$ Solution (Life Technologies, Carlsbad, USA) according to the producer's standard. DNA was removed from the isolated RNA samples by DNase using DNA-free TM reagent (Life Technologies). Two micrograms of RNA were reverse transcribed to cDNA with the High Capacity cDNA Reverse Transcription Kit (ThermoFisher Scientific, Waltham, USA). Expression of the PIM2 gene was assessed by TaqMan RQ-PCR assay using predesigned TaqMan Gene Expression Assays Hs00179139_m1 for PIM2), on a 7500

Table 1. Clinical data of patients with CLL and the control group

\begin{tabular}{|l|c|c|}
\multicolumn{1}{|c|}{ Parameters } & Patients with CLL & Control group \\
\hline$n$ & 67 & 14 \\
\hline Age (median) & $66(49-90)$ & $59(47-88)$ \\
\hline Sex & & \\
F/M & $35 / 32$ & $7 / 7$ \\
\hline CR/NCR & $32 / 35$ & NA \\
\hline Rai stage & & \\
0 & 0 & \\
I & 18 & NA \\
II & 24 & \\
III & 9 & \\
IV & 16 & \\
LDT & & \\
$\geq 12$ months & 20 & \\
$<12$ months & 47 & \\
\hline
\end{tabular}

$\mathrm{CLL}$ - chronic lymphocytic leukemia; F - females; $\mathrm{M}$ - males; $\mathrm{CR}$ - complete remission; NCR - no complete remission; LDT - lymphocyte doubling time; NA - not applicable. 

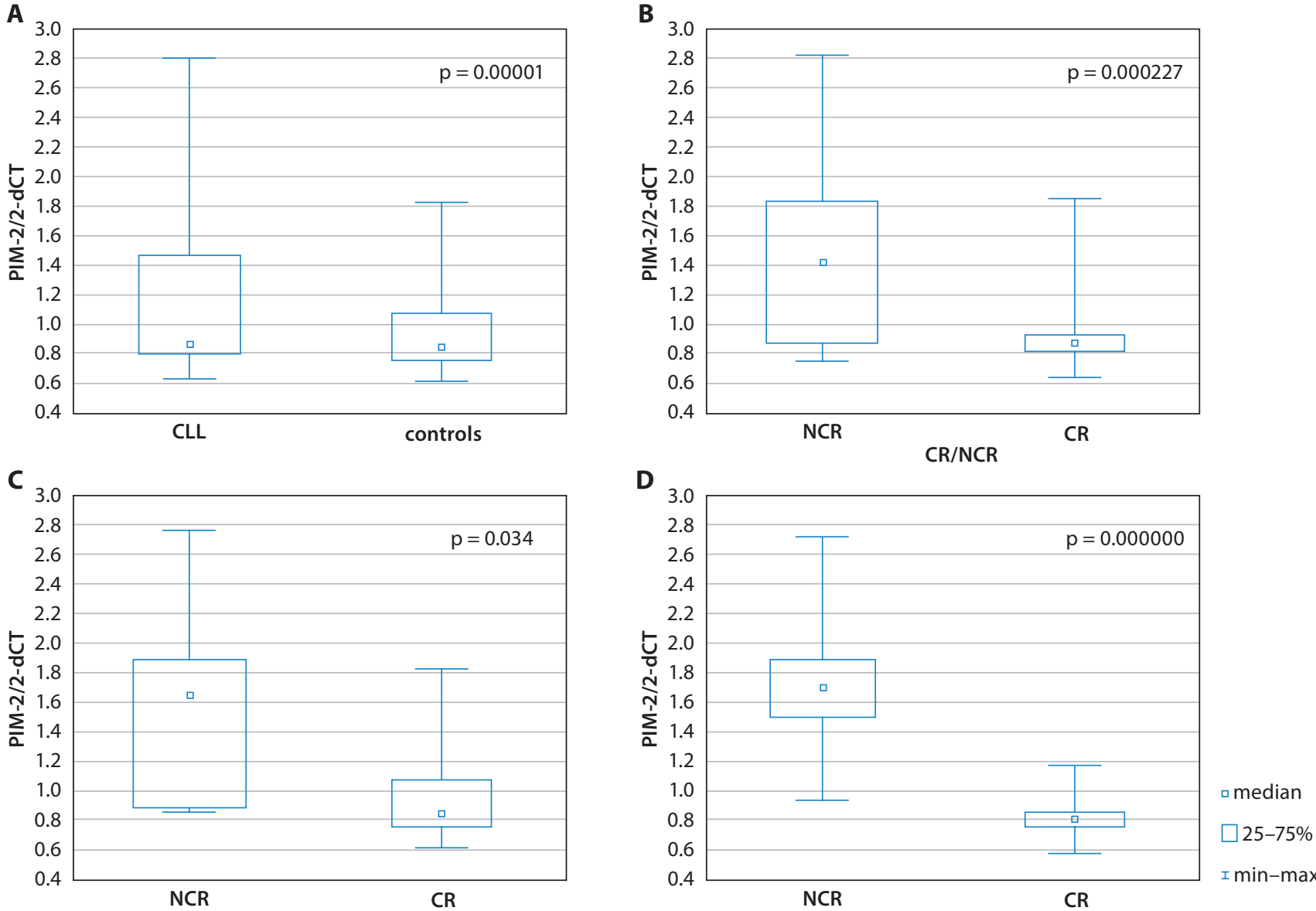

Fig. 1. Comparison of median PIM2 expression between patients with CLL and healthy controls (A); between patients who achieved CR vs NCR (B); between patients who reached CR vs NCR after FC-R treatment (C) and patients with LDT $<12$ months (a) and $\geq 12$ months (b) (D)

RQ-PCR System (Applied Biosystems, Foster City, USA). The $\beta$-glucuronidase gene (GUS) was used as a control gene (TaqMan Gene Expression Assay: Hs99999908_m1). The relative gene expression level was measured as the difference between the $\mathrm{C}_{\mathrm{t}}$ values of the PIM2 gene and the GUS gene $\left(\Delta C_{t}\right)$ and expressed as $2^{-\Delta C t}$.

\section{Western blot}

Cells were lysed using radioimmunoprecipitation assay (RIPA) buffer and centrifuged at 10,000 $\times$ g, $10 \mathrm{~min}, 4^{\circ} \mathrm{C}$. Clear supernatant was collected and the protein concentration was determined using a bicinchoninic acid protein assay kit (Sigma-Aldrich, St. Louis, USA). Cell lysates (40 $\mu$ g per line) after separation by sodium dodecyl sulfate-polyacrylamide gel electrophoresis (SDS-PAGE) in 12\% gel were transferred to nitrocellulose (Bio-Rad, Hercules, USA), blocked for $1 \mathrm{~h}$ with $5 \%$ fat-free dry milk in tris-buffered saline (TBS) and incubated overnight at $4^{\circ} \mathrm{C}$ with the appropriate primary antibody. The following primary antibodies were used: rabbit monoclonal antibody against PIM2 (Cell Signaling Technology, Leiden, the Netherlands) and rabbit polyclonal antibody against $\beta$-actin (Abcam, Cambridge, UK). Immunodetection was performed using secondary horseradish peroxidase (HRP)-conjugated goat anti-rabbit immunoglobulins
(Dako, Glostrup, Denmark). After washing, the membrane was briefly incubated using Lumi-Light ${ }^{\text {PLUS }}$ western blot substrate (Roche, Basel, Switzerland) and exposed to BioLight film (Eastman Kodak, Rochester, USA). The films were photographed using a Canon Power Shot A640 camera and analyzed with ImageJ $1.45 \mathrm{~S}$ software (Wayne Rasband, National Institutes of Health, USA) using $\beta$-actin as a control. The integrated density was calculated for each line.

\section{Statistical analysis}

Statistical analysis was performed using the non-parametric Mann-Whitney U test for independent samples. The correlation between quantitative variables was tested with Spearman's rank correlation test. Progression-free survival (PFS) and time to first treatment (TTFT) analysis was performed using the Wilcoxon test. The result was considered statistically significant when $\mathrm{p} \leq 0.05$.

\section{Results}

Median PIM2 gene expression in leukemic cells of CLL patients was higher than in peripheral blood lymphocytes of the control group: $0.88(0.63-2.81)$ vs $0.73(0.63-0.86)$, 

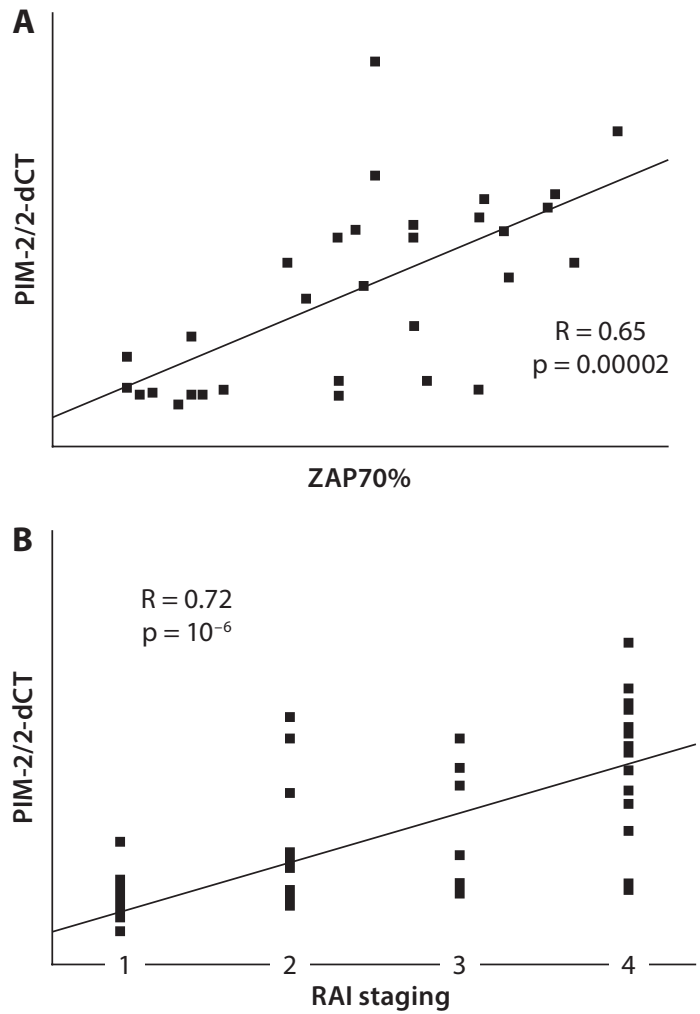

Fig. 2. Positive correlation between PIM2 gene expression and the percentage of malignant lymphocytes with ZAP70 expression, $R=0.69$, $p=0.00002$ (A) and between PIM2 gene expression and Rai stage, $R=0.72, p=0.00000(B)$

$\mathrm{p}=0.000001$. Chronic lymphocytic leukemia patients with LDT shorter than $<12$ months showed higher PIM2 expression than patients with LDT $\geq 12$ months: 1.79 (0.95-2.81) vs $0.85(0.63-1.25), \mathrm{p}=0.00000$ (Fig. 1A,D). Notably, a positive correlation between PIM2 gene expression and the percentage of malignant lymphocytes with ZAP70 expression and the Rai stage was revealed: $R=0.69, p=0.00002$ and $\mathrm{R}=0.72, \mathrm{p}=0.00000$, respectively (Fig. 2A,B). Chronic lymphocytic leukemia patients with high expression levels of the PIM2 gene (above the median) had shorter PFS than patients with low PIM2 expression, 11 months (4-38) and 22 (5-60), respectively, $\mathrm{p}=0.01$ (Fig. 3A). Moreover, TTFT was longer in patients with PIM2 expression below median than in patients with higher PIM2 expression, 90 days (30-180) and $30(30-180)$ respectively, $\mathrm{p}=0.0003$ (Fig. 3B). In addition, it was found that patients who achieved CR had significantly lower expression of the PIM2 gene than patients with no CR (NCR): $0.84(0.63-1.84)$ vs 1.41 (0.74-2.81), $\mathrm{p}=0.000227$ (Fig. 1B). PIM2 expression in peripheral blood lymphocytes of CLL patients in CR was lower than in leukemic lymphocytes of those patients analyzed before the treatment, and was comparable to healthy controls (data not shown). Notably, we found that in patients with CR after R-FC treatment, median PIM2 expression was lower than in patients with NCR, $0.086(0.83-1.84)$ and 1.67 (0.88-2.81), respectively, $\mathrm{p}=0.034$ (Fig. $1 \mathrm{C}$ ). To confirm the results at the PIM2, mRNA level the cell lysates from 9
CLL patients were analyzed by western blot. Protein bands were found in all analyzed cell lysates. The number of bands differed between the samples, which reflected the presence of different isoforms of the protein (Fig. 4A,B). As expected, the densitometric analysis revealed high PIM2 expression in CLL patients when compared with the controls (Fig. 4C). Moreover, a positive correlation between $\mathrm{mRNA}$ and protein level was found, $R=0.98, p=0.000002$ (data not shown).

\section{Discussion}

PIM (1, 2 and 3) (provirus integration site for Moloney murine leukemia virus) family proteins are highly conserved serine/threonine kinases, which are considered to be involved in cancer progression and the development of resistance to chemotherapeutic agents. Their elevated expression has been observed in solid tumors and hematological malignancies. ${ }^{6,13}$ The activation of PIM kinases modulates a broad range of cellular phenomena, including apoptosis, progression of the cell cycle, differentiation, protein translation, and interactions with

A

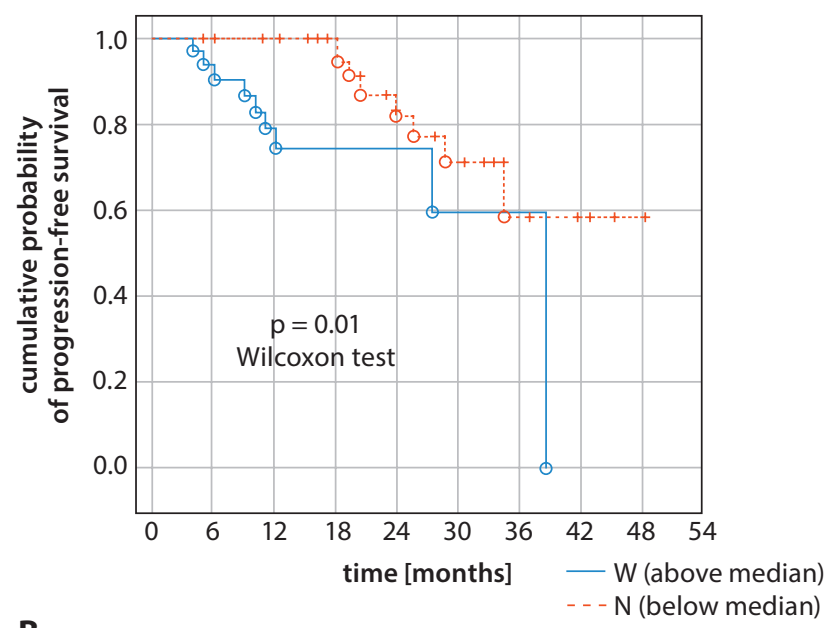

B

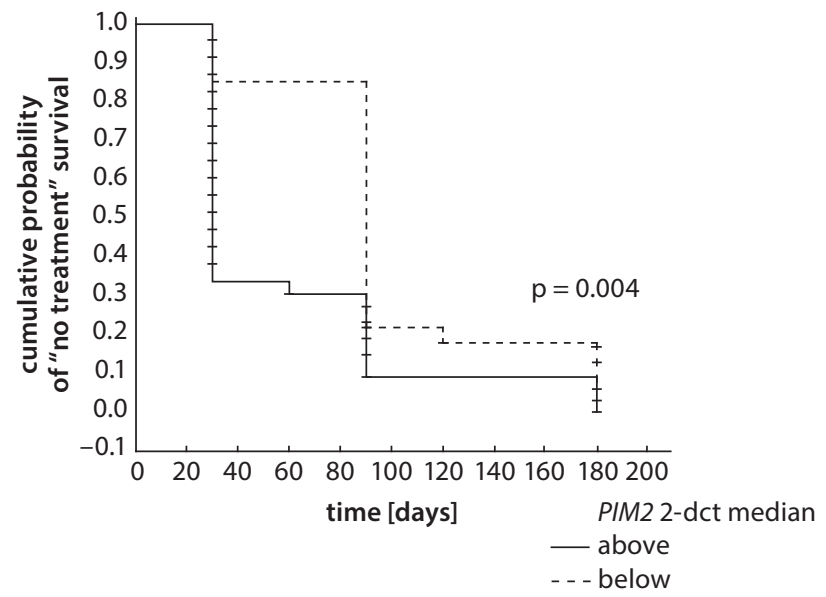

Fig. 3. Kaplan-Meier PFS (A) and TTFT (B) curves with regard to PIM2 gene expression in CLL patients stratified as above or below the median value of PIM2 gene expression (0.88) 


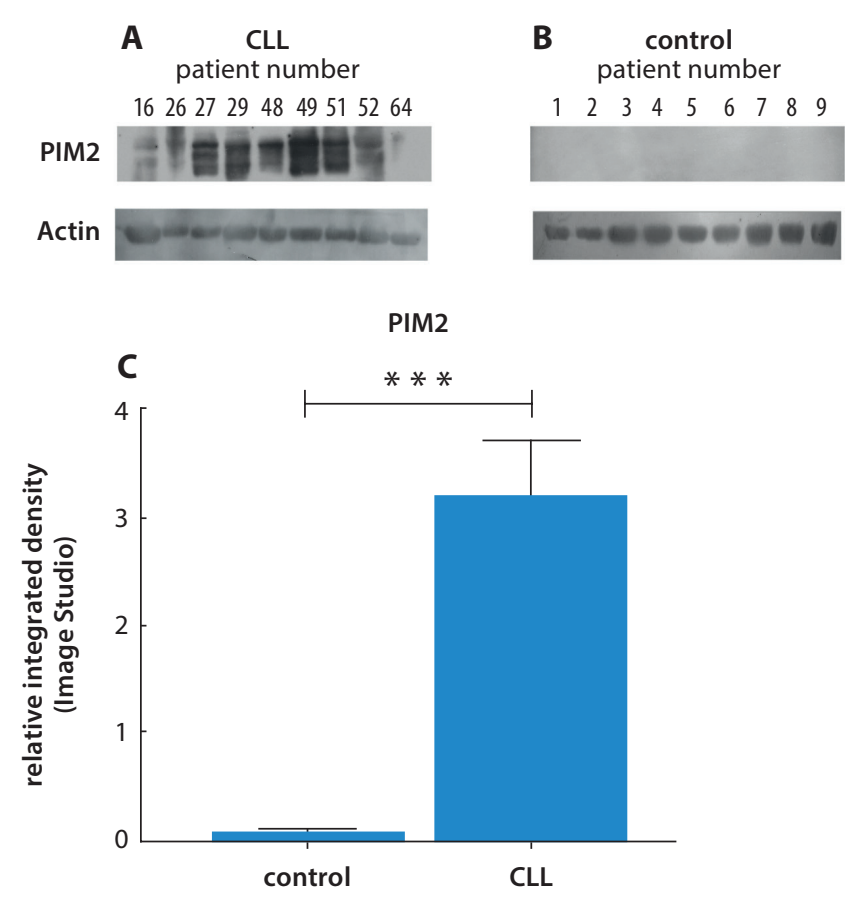

Fig. 4. Western blot analysis of PIM2 in CLL patients and normal controls $(A, B)$, densitometric analysis of PIM2 expression in mononuclear cells of CLL patients and normal controls, ${ }^{* *} p<0.0001$ (C)

the microenvironment. ${ }^{5}$ Their expression is mediated by JAK-STAT and NFKB signaling pathways after the stimulation of growth factors, cytokines and TNF family receptors. ${ }^{14}$ These kinases have also been demonstrated to play a role in the development of hematological malignancies, since their expression was found in both cell lines and malignant cells obtained from patients with different myeloid and lymphoid malignancies. ${ }^{5,15}$

The inhibition of mechanisms leading to in vivo apoptosis is considered as one of the most important factors leading to the accumulation of leukemic lymphocytes of patients with chronic lymphocytic leukemia. Extensive research conducted on this topic has led to the identification of intracellular phenomena involved in the prolonged lifespan of CLL lymphocytes, such as the BCR-induced pathway and antiapoptotic protein BCL-2 hyperexpression. ${ }^{16}$ However, our knowledge of the mechanisms responsible for the inhibition of apoptosis in CLL cells needs to be extended, as well as the research of factors which would predict the risk of rapid progression of this disease in order to single out the patients who may benefit from early institution of the treatment.

The results of some studies published so far strongly suggest that PIM kinases act as survival-promoting factors for CLL cells, since their inhibition by an imidazo [1,2-b] pyridazine small molecule, SGI-1776, led to the in vitro apoptosis of those cells accompanied by reduction of the MCL1 gene transcript. ${ }^{17}$ Recently, several pan-PIM kinase inhibitors were developed. ${ }^{18,19}$ The antiapoptotic effect of the inhibition of PIM kinases by 3 different inhibitors was also demonstrated by Decker et al. ${ }^{7}$
PIM2 kinase attracted particular attention for the research on the biology of CLL cells. The serine/threonine kinase PIM2 is an oncogenic factor that has been shown to promote cell survival by activating the expression of $N F_{\mathrm{K}} B$-dependent gene expression following a number of proliferation-inducing signals. ${ }^{4,20,21}$ As demonstrated by knockdown experiments, this kinase acts in CLL cells as a survival-promoting factor, but in contrast to PIM1, it does not affect the surface expression of the CXCR4 receptor. ${ }^{7}$ Increased expression of the PIM2 gene as compared with normal lymphocytes was reported by several authors. ${ }^{7,10,22}$ Cohen et al. found that higher expression of this gene was correlated with such negative prognostic factors as shorter lymphocyte doubling time and higher Binet stage. ${ }^{10}$ Moreover, Hüttmann et al. demonstrated that PIM2 mRNA expression ratio in highly purified CLL lymphocytes, when calculated using unpurified mononuclear cells of healthy donors as a template, was higher not only in advanced Rai stages, but also in CD38 ${ }^{+} / \mathrm{ZAP}^{+} 0^{+}$ cells as compared to double-negative ones. ${ }^{22}$ In contrast, Decker et al. did not find a relationship between PIM transcript levels or PIM protein expression levels and such risk factors in CLL as clinical stage or IgVH mutational status. ${ }^{7}$

Given the biological function of PIM2 kinase as a survival-promoting factor and the above-quoted findings which suggest a possible relationship between its elevated expression and some prognostically unfavorable clinical and biological factors of CLL, we decided to investigate whether expression of the PIM2 gene is also related to a more aggressive course of the disease and whether it has an impact on the response to cytostatic treatment.

In line with previous studies, we found that PIM2 mRNA content in circulating CLL lymphocytes was higher than in normal peripheral blood lymphocytes obtained from healthy donors. We also demonstrated that increased expression of the PIM2 gene was related to shorter LDT, more advanced clinical stage and higher percentage of ZAP70-positive cells. Western blot analysis of PIM2 protein content in 9 representative samples demonstrated a correlation between mRNA and protein content, which suggests transcriptional regulation of expression of this factor. An original aspect of our study is the finding that high PIM2 expression was associated with a short TTFT and short PFS after the successful frontline treatment. This correlation between high PIM2 expression and aggressive clinical course of CLL is consistent with the relationship between expression of this kinase and the abovementioned negative prognostic factors. It therefore remains to be determined whether this negative prognostic value of PIM2 expression is independent or related to other factors known to negatively influence the clinical course of CLL.

We also found that the CR rate was lower in patients who strongly express the PIM2 gene, as compared to low expressors of the gene in question. This negative predictive value of high expression of the PIM2 gene was found 
both for the whole group of patients who underwent immunochemotherapy and for the subgroup in whom a CR was obtained using the FC-R regimen. To our knowledge, this is the first observation published so far concerning the impact of expression of the PIM2 gene in CLL cells on the results of immunochemotherapy. Our findings may suggest that PIM2 decreases the sensitivity of CLL cells to purine analogue-based immunochemotherapy. It is thus possible that PIM2 kinase survival-promoting activity compensates the apoptosis pathway triggered by purine analogues, alkylating drugs and rituximab. It is noteworthy that the overexpression of PIM kinases has been reported to confer resistance to rapamycin, an mTOR (mammalian target of rapamycin) inhibitor, and another drug active in hematological malignancies. ${ }^{23,24}$

Our findings warrant further studies on the value of expression of the PIM2 gene as a prognostic factor associated with a more aggressive clinical course of CLL, and a predictive factor of the response to immunochemotherapy administered as frontline treatment. These findings also support the idea of studying the PIM kinases as therapeutic targets in lymphoid malignancies.

\section{References}

1. Breuer ML, Cuypers HT, Berns A. Evidence for the involvement of Pim-2, a new common proviral insertion site, in progression of lymphomas. EMBO J. 1989;8(3):743-748.

2. Baytel D, Shalom S, Madgar I, et al. The human Pim-2 proto-oncogene and its testicular expression. Biochim Biophys Acta. 1998;1442 (2-3):274-285.

3. Mikkers $\mathrm{H}$, Allen J, Knipscheer $\mathrm{P}$, et al. High-throughput retroviral tagging to identify components of specific signaling pathways in cancer. Nat Genet. 2002;32(1):153-159. Erratum in: Nat Genet. 2002;32(3):459.

4. Fox CJ, Hammerman PS, Cinalli RM, et al. The serine/threonine kinase Pim-2 is a transcriptionally regulated apoptotic inhibitor. Genes Dev. 2003;17(15):1841-1854.

5. Nawijn MC, Alendar A, Berns A. For better or for worse: The role of Pim oncogenes in tumorigenesis. Nat Rev Cancer. 2011;11:23-34.

6. Kapelko-Słowik K, Owczarek TB, Grzymajło K, et al. Elevated PIM2 gene expression is associated with poor survival of patients with acute myeloid leukemia. Leuk Lymphoma. 2016;57(9):2140-2149.

7. Decker S, Finter J, Forde AJ, et al. PIM kinases are essential for chronic lymphocytic leukemia cell survival (PIM2/3) and CXCR4-mediated microenvironmental interactions (PIM1). Mol Cancer Ther. 2014;13:1231-1245.
8. Martín-Sánchez E, Odqvist L, Rodríguez-Pinilla SM, et al. PIM kinases as potential therapeutic targets in a subset of peripheral T cell lymphoma cases. PLOS ONE. 2014;9:e112148.

9. Asano J, Nakano A, Oda A, et al. The serine/threonine kinase Pim-2 is a novel anti-apoptotic mediator in myeloma cells. Leukemia. 2011;25:1182-1188.

10. Cohen AM, Grinblat B, Bessler $\mathrm{H}$, et al. Increased expression of the hPim-2 gene in human chronic lymphocytic leukemia and non-Hodgkin lymphoma. Leuk Lymphoma. 2004;45(5):951-955.

11. Hallek M, Cheson BD, Catovsky D, et al. Guidelines for the diagnosis and treatment of chronic lymphocytic leukemia: A report from the International Workshop on Chronic Lymphocytic Leukemia updating the National Cancer Institute-Working Group 1996 guidelines. Blood. 2008;111:5446-5456.

12. Eichhorst B, Robak T, Montserrat E, et al. ESMO Guidelines Committee. Chronic lymphocytic leukaemia: ESMO Clinical Practice Guidelines for diagnosis, treatment and follow-up. Ann Oncol. 2015;26(Suppl 5):v78-84.

13. Narlik-Grassow M, Blanco-Aparicio C, Carnero A. The PIM family of serine/threonine kinases in cancer. Med Res Rev. 2014;34:136-159.

14. Brault L, Gasser C, Bracher F, et al. PIM serine/threonine kinases in the pathogenesis and therapy of hematologic malignancies and solid cancers. Haematologica. 2010;95(6):1004-1015.

15. Kapelko-Słowik K, Urbaniak-Kujda D, Wołowiec D, et al. Expression of PIM-2 and NF-KB genes is increased in patients with acute myeloid leukemia (AML) and acute lymphoblastic leukemia (ALL) and is associated with complete remission rate and overall survival. Postepy Hig Med Dosw. 2013;67:553-559.

16. Tam CS, Seymour JF, Roberts AW. Progress in BCL2 inhibition for patients with chronic lymphocytic leukemia. Semin Oncol. 2016;43(2):274-279.

17. Chen LS, Redkar S, Bearss D, et al. Pim kinase inhibitor, SGI1776 , induces apoptosis in chronic lymphocytic leukemia cells. Blood. 2009;114(19):4150-4157.

18. Gómez-Abad C, Pisonero H, Blanco-Aparicio C, et al. PIM2 inhibition as a rational therapeutic approach in B-cell lymphoma. Blood. 2011;118:5517-5527.

19. Garcia PD, Langowski JL, Wang Y, et al. Pan-PIM kinase inhibition provides a novel therapy for treating hematologic cancers. Clin Cancer Res. 2014;20:1834-1845.

20. Hammerman PS, Fox CJ, Birnbaum MJ, et al. Pim and Akt oncogenes are independent regulators of haematopoietic cell growth and survival. Blood. 2005;105(11):4477-4483.

21. Hammerman PS, Fox C, Cinalli R, et al. Lymphocyte transformation by Pim-2 is dependent on nuclear factor-kB activation. Cancer Res. 2004;64:8341-8348.

22. Hüttmann A, Klein-Hitpass $L$, Thomale J, et al. Gene expression signatures separate B-cell chronic lymphocytic leukaemia prognostic subgroups defined by ZAP-70 and CD38 expression status. Leukemia. 2006;20:1774-1782.

23. Swords R, Kelly K, Carew J, et al. The Pim kinases: New targets for drug development. Curr Drug Targets. 2011;12(14):2059-2066.

24. Tamburini J, Green AS, Bardet V, et al. Protein synthesis is resistant to rapamycin and constitutes a promising therapeutic target in acute myeloid leukemia. Blood. 2009;114(8):1618-1627. 